\title{
Torsional Characteristics of Single Walled Carbon Nanotube with Water Interactions by Using Molecular Dynamics Simulation
}

\author{
V. Vijayaraghavan, C. H. Wong*
}

(Received 30 January 2014; accepted 24 March 2014; published online July 1, 2014)

\begin{abstract}
The torsional characteristics of single walled carbon nanotube (SWCNT) with water interactions are studied in this work using molecular dynamics simulation method. The torsional properties of carbon nanotubes (CNTs) in a hydrodynamic environment such as water are critical for its key role in determining the lifetime and stability of CNT based nano-fluidic devices. The effect of chirality, defects and the density of water encapsulation is studied by subjecting the SWCNT to torsion. The findings show that the torsional strength of SWCNT decreases due to interaction of water molecules and presence of defects in the SWCNT. Additionally, for the case of water molecules encapsulated inside SWCNT, the torsional response depends on the density of packing of water molecules. Our findings and conclusions obtained from this paper is expected to further compliment the potential applications of CNTs as promising candidates for applications in nano-biological and nano-fluidic devices.
\end{abstract}

Keywords: Carbon nanotube; Water interaction; Torsion; Water encapsulation; Nano-fluid; Molecular dynamics

Citation: V. Vijayaraghavan and C. H. Wong, "Torsional Characteristics of Single Walled Carbon Nanotube with Water Interactions by Using Molecular Dynamics Simulation", Nano-Micro Lett. 6(3), 268-279 (2014). http://dx.doi.org/10.5101/nml140029a

\section{Introduction}

Research in carbon nanotubes (CNTs) has gained enormous interest in nanotechnology and materials science due to its remarkable physical and mechanical properties $[1,2]$. CNTs possess a unique combination of high strength and ultra-light weight which makes it a promising application in nanoelectromechanical systems (NEMS) such as springs [3], and oscillators [4]. These applications require a critical understanding of the torsional properties of CNTs. In addition, CNTs have also demonstrated the potential to be used in the field of nano-drug delivery systems $[5,6]$, and nanofluidic devices $[7,8]$. Hence, understanding the torsional elastic properties of CNTs in a hydrodynamic environment is important to optimize the performance of CNTs for its use in NEMS and nano-level biological devices.

Many studies have been aimed to investigate the torsional elastic properties of CNTs by means of experimental or theoretical based approaches [9-12]. Lu and $\mathrm{Hu}[13]$ proposed a novel and improved finite element 3D model based on molecular mechanics approach to study the elastic properties of CNTs under tension and torsion. Using this model, they investigated the effects of diameter and helicity on the Young's modulus and shear modulus of SWCNTs. Wang et al [10] deployed Molecular dynamics (MD) simulation technique to determine the mechanical properties of CNTs under torsion. They found that the shear modulus of CNT increases with increasing the radius of CNT. Zhang et al [12] studied the effect of various types of defects and temperature on the torsional responses of

School of Mechanical and Aerospace Engineering, Nanyang Technological University, 50 Nanyang Avenue, Singapore 639798

*Corresponding author. E-mail: chwong@ntu.edu.sg 
defective SWCNT. The studies showed that the torsional strength is strongly dependent on the chirality, type of defects, and temperature. Faria et al [14] studied the elastic behavior of chiral SWCNTs namely the $(6,3) \mathrm{CNT}$, under combined tension-twisting. They conducted MD simulation studies on the SWCNT subjected to three forms of mechanical loading, viz. pure tension, pure torsion and combined tension-torsion. It was found that the elastic modulus of the SWCNT under pure tension and torsion were much higher than that of the combined tension-torsion. Jeong et al [15] studied the torsional responses of carbon nanotubes filled with SWCNTs, fullerenes and gases. Their investigations revealed that the torsional responses of SWCNTs will be distinct which depends on the density and nature of the filling material. In addition, the torsional characteristics of CNTs filled with metals such as copper [16] and silicon atoms [17] have also been studied in the literature.

It can be seen from the above mentioned literature studies that the torsional properties of the CNTs have been well studied and investigated in freeform and those filled with gases and metal atoms. However, it should be noted that the torsional strength of CNTs in hydrodynamic environment (with water) is still yet to be investigated. It is widely recognized that CNTs operating in hydrodynamics environment (such as nano-fluidic devices) will experience distinct loading conditions due to interaction of the surrounding water molecules. Hence, the main objective of our work is to investigate the torsional characteristics of free form and water submerged SWCNT. Furthermore, the influence of geometrical characteristics such as the chirality and the effect of defects on the torsional response of a CNT in water medium present an important and crucial design input for fabricating CNT based nano-fluidics and NEMS devices. The elastic properties of SWCNTs under various forms of water interaction have been comprehensively studied and the results are presented in this paper.

\section{Computational model}

The numerical simulations described in this work were carried out by using the classical molecular dynamics simulation method using LAMMPS software [18] where force field equations are used to describe the inter-atomic interactions. These inter-atomic interactions can be divided into the interaction between the carbon atoms of SWCNT, the interaction between the water molecules and the carbon atoms of SWCNT and the interaction between the water molecules. The interatomic interactions of carbon atoms in SWCNT are described using the Adaptive Intermolecular Reactive Empirical Bond Order (AIREBO) potential [19]. The AIREBO potential is able to accurately describe the properties of solid-state and molecular carbon nanostructures [20,21] while maintaining the accuracies of the $a b$ initio and semi-empirical methods in simulating large systems [22]. The torsional interaction due to dihedral angles in the system is also implemented in addition to the long range interactions in the AIREBO potential. The AIREBO function is defined mathematically as [19],

$$
E_{A I R E B O}=E_{R E B O}+E_{L J}+E_{T O R S}
$$

where the $E_{R E B O}$ represents the interaction energy defined by the Brenner's second generation REBO potential [23]. The torsional interaction is represented by $E_{T O R S}$ and $E_{L J}$ describes the long range Lennard-Jones (LJ) (12-6) interaction between the carbon atoms.

The non-bonded interactions between the SWCNTs and the water molecule is typically modeled using a LJ potential function $[24,25]$, which accurately describes the short-range electron repulsion and long-range electron attraction between the CNT and water molecules. The LJ potential is tuned using the parameters obtained from Ref. $[26,27]$ to accurately describe the experimentally observed water-graphene contact angle. The non-bonded interactions between the carbon atoms and the water molecule is given by [26],

$$
E_{n o n-b o n d}=\sum_{i, j} 4 \varepsilon\left[\left(\frac{\sigma_{i j}}{r_{i j}}\right)^{12}-\left(\frac{\sigma_{i j}}{r_{i j}}\right)^{6}\right]
$$

where $\varepsilon_{C O}=0.392 \mathrm{~kJ} / \mathrm{mol}$ and $\sigma_{C O}=0.319 \mathrm{~nm}$ are the LJ parameters between carbon to oxygen atoms [26]. Similarly, $\varepsilon_{C H}=0.243 \mathrm{~kJ} / \mathrm{mol}$ and $\sigma_{C H}=0.282$ $\mathrm{nm}$ are the LJ parameters between carbon to hydrogen atoms [27].

The interaction between the water molecules is described using the flexible fixed-point charge (FPC) water model [28]. The FPC water model is capable of accurately reproducing the properties of water for different ranges of pressure, temperature, integration time steps and system sizes. The FPC water model is described as [28],

$$
E F P C=E_{\text {bond }}+E_{\text {bend }}+E_{v d W}+E_{\text {els }},
$$

where $E_{b o n d}$ and $E_{b e n d}$ are intra-molecular potential functions that represent the bond strength energy and bending energy in a water molecule respectively. The terms $E_{e l s}$ and $E_{v d w}$ denote the electrostatic potential and van der Waals potential between water molecules respectively. The complete details of this potential function are described in the work by Zhang et al. [28].

\section{Calculation of torsional force and torque on SWCNT}

The torsional force is calculated by summation of inter-atomic forces between carbon atoms at the ends 
of the SWCNT. The inter-atomic force, $\vec{F}_{i j}$ between atoms $i$ and $j$ is obtained by the gradient of potential energy described mathematically as [18],

$$
\vec{F}_{i j}=-\left(\frac{d E\left(r_{i j}\right)}{d r_{i j}}\right)
$$

where $r_{i j}$ is the distance between the atoms $i$ and $j$ and $E\left(r_{i j}\right)$ is the potential between the atoms $i$ and $j$.

The torque, $T$ of SWCNT is then calculated by the product of torsional force $F$ and the radius $R$ of the SWCNT [12].

\section{Simulation details}

The work described in this paper is divided into two parts. The first part of the paper will focus on the torsional elastic characteristics of uncapped (open ended) SWCNTs submerged in water. The elastic properties of the capped SWCNTs encapsulated with varying densities of water molecules are discussed in following. Figure 1 describes the method of torsional loading condition applied in SWCNT considered in our study. To investigate the effect of geometry on the torsional properties of SWCNT, we considered armchair and zigzag SWCNTs of varying aspect ratios. Furthermore, the effect of defect density and distribution on the torsional properties is also investigated in this paper. In order to accomplish this, firstly we constructed a single vacancy defect approximately at the center of the SWCNT. This vacancy defect is then expanded in the radial or axial direction to form a bigger defect as illustrated in Fig. 2. This method of defect reconstruction allows us to study the role of defect distribution on the torsional properties of SWCNT [29,30].

The computational procedure described herein is similar to our previous work on the tensile loading characteristics of SWCNTs in water [30]. A simulation box of dimension $40 \AA \times 40 \AA \times 150 \AA$ is deployed in this work to simulate the interaction of CNT with the water molecules and PBCs are applied on all three directions of the system. Each time step in the MD simulation has a unit of $1 \mathrm{fs}$. At the beginning of the simulation, we equilibrate the system to release the residual stresses by achieving thermal equilibrium in an NVT ensemble. The simulations are carried out by maintaining a constant system temperature of $300 \mathrm{~K}$ and the density of water molecules is at $0.997 \mathrm{~g} / \mathrm{cm}^{3}$ (approx. $1 \mathrm{~g} / \mathrm{cm}^{3}$ ). The temperature stability of the system is achieved by employing Nose-Hoover thermostat [31,32]. Following this, the torsional loading is applied on the SWCNT as defined in Fig. 1. Equal number of atoms is fixed on the either ends of SWCNT and the number of fixed atoms at each end of SWCNTs for different geometries is listed in Table 1. The method of torsional rotation applied in our simulation is shown in Fig. 1, where the atoms at one end of the SWCNT are rigidly fixed (enclosed inside red rectangle) and the atoms at the other end (enclosed inside black rectangle) are subjected to constant incremental torsional rotation of 0.006 radians. After each rotation, the remaining atoms (those that are not fixed) are subjected to equilibration for

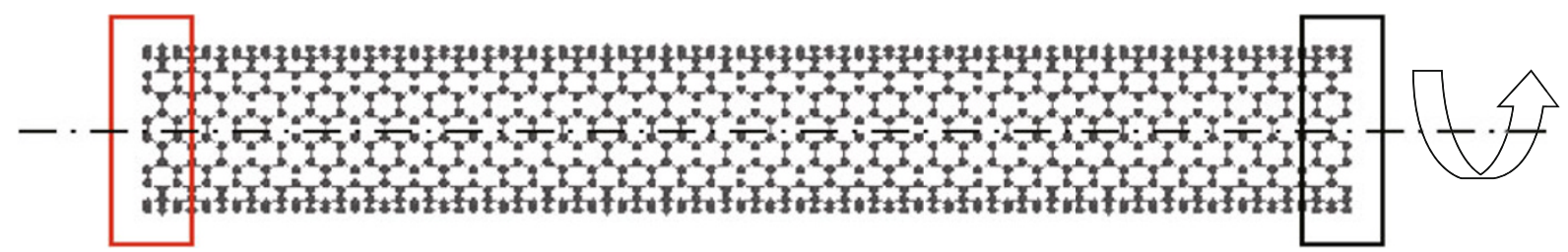

Fig. 1 The procedure of torsional loading of the SWCNT in our study. The end atoms enclosed within the red rectangle are fixed rigidly. The atoms enclosed inside the black rectangle is subjected to clockwise rotational displacement (twist speed $=$ $0.0062 \mathrm{rad} / \mathrm{ps}$, i.e. strain rate $=0.001 \mathrm{ps}^{-1}$ ) to effect torsion.

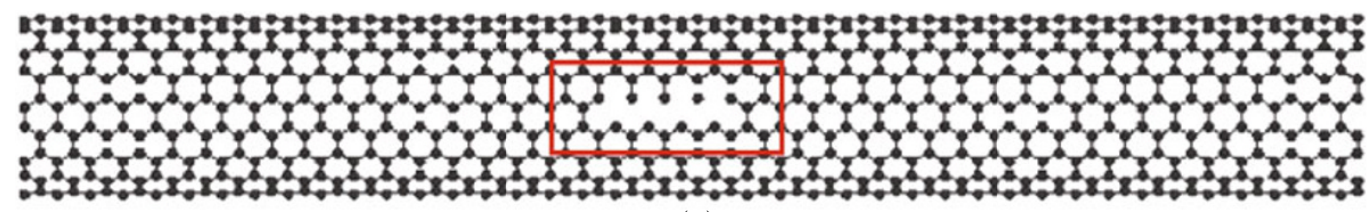

(a)

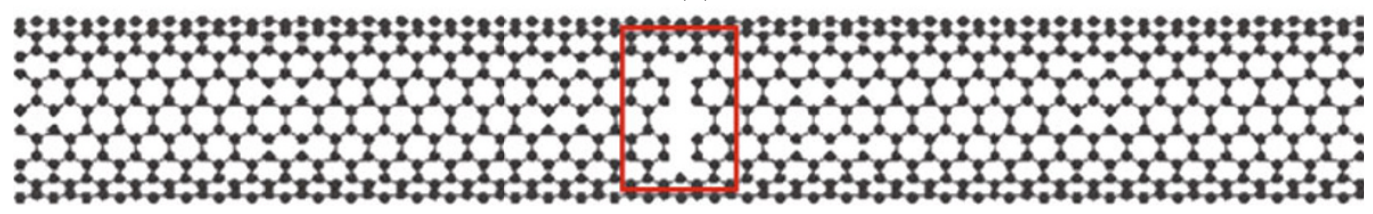

(b)

Fig. 2 An illustration of vacancy defect construction in a perfect SWCNT lattice is employed in our study. A single vacancy defect is constructed at the center and the defect is expanded along the (a) axial or (b) radial direction. Only the front part of the SWCNT is shown. 
Table 1 Number of Carbon atoms fixed in each end of SWCNTs

\begin{tabular}{cc}
\hline Geometry of SWCNT & $\begin{array}{c}\text { Number of carbon atoms fixed } \\
\text { in either ends of SWCNT }\end{array}$ \\
\hline$(10,10)$ & 164 \\
$(5,5)$ & 82 \\
$(18,0)$ & 176 \\
$(10,0)$ & 88 \\
\hline
\end{tabular}

1000 time steps (1 ps) such that the atoms attain the favorable minimum energy positions. The twist speed applied to the movable fixed end of carbon atoms in SWCNT in our MD simulation is $0.006 \mathrm{rad} / \mathrm{ps}$. The end atoms are subsequently again twisted to a new position after calculation of the trajectories of atoms based on Verlet algorithm [33]. The remaining atoms are relaxed in an NVT ensemble and the procedure is repeated until failure occurs.

The snap shot of the equilibrated system consisting of a $(10,0)$ SWCNT in a simulation box filled with water molecules of density $0.9 \mathrm{~g} / \mathrm{cm}^{3}$ is depicted in Fig. 3(a). We note from this figure that the narrow diameter of $(10,0)$ SWCNT allows for a single-file arrangement of water molecules inside the SWCNT, which is in agreement with results reported in Ref. [34]. The radial distribution function (RDF) of water molecules from the center of the $(10,0)$ SWCNT given by $\mathrm{g}\left(R_{d}\right)$, where $R_{d}$ is the radial distance from the center of the SWCNT is shown in Fig. 3(b). It can be observed from this figure that the first peak occurs at radial distance less than $0.05 \mathrm{~nm}$ which indicates a region near the center of the SWCNT. This means that it is more likely to find a water molecule at the center than any other region inside the SWCNT. The radial distribution then drops down to zero and remains at this value until radial distances exceed $0.69 \mathrm{~nm}$. This is due to the van der Waal's interaction between the water molecules and the carbon atoms of SWCNT as described by Eqn. (2). A second characteristic peak is observed outside the surface of SWCNT which is in qualitative agreement with the previous simulated results described in Ref. [35]. At larger distances from the surface of the $\mathrm{CNT}$, the radial distribution function fluctuates around the value of 1 due to weak interaction between the water molecules and the carbon atom. Overall, the resulting post-equilibrated structure of SWCNT submerged in simulation box of water molecules helps us to confirm the accuracy of the simulation.

\section{Results and discussion}

\section{Validation of the simulation model}

The elastic properties of a free form SWCNT under torsional loading is discussed in this section. In order

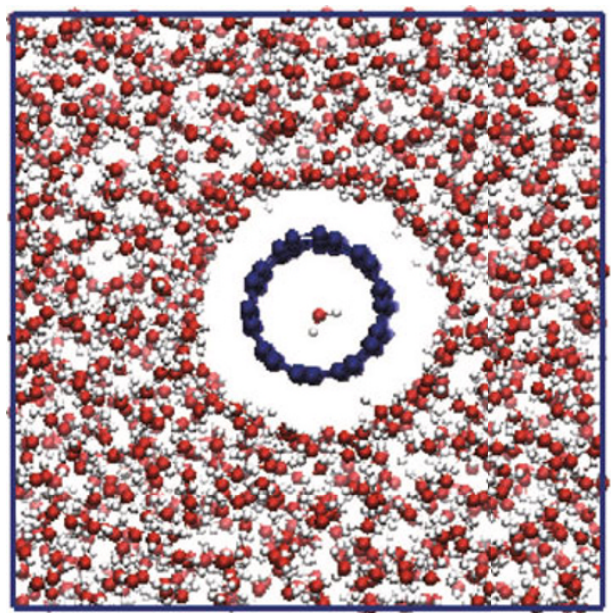

(a)

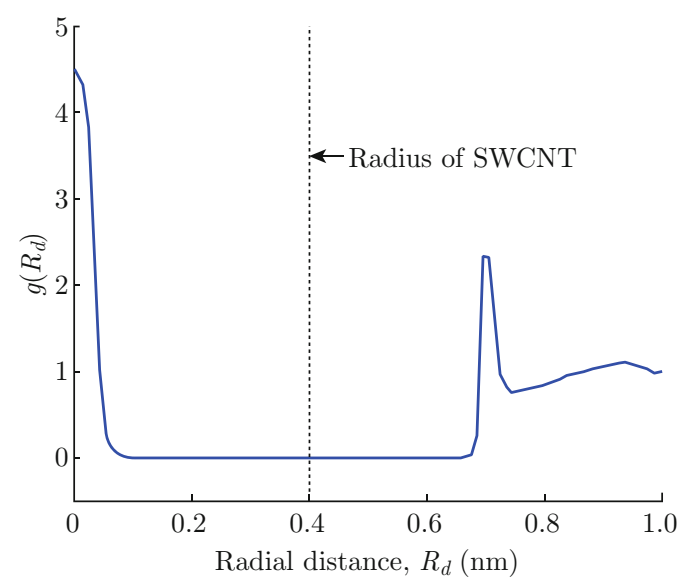

(b)

Fig. 3 Snap shot of an equilibrated structure of SWCNT submerged in water where the carbon atoms of CNT are depicted by the blue balls and the water molecules represented by red and white balls (a) and (b) radial distribution of water molecules from the center of the SWCNT.

to validate the computational model employed in the current study, we subjected the same SWCNT model (a (10, 10) SWCNT of length $100 \AA$ ) as used in Ref. [15] simulated using the AIREBO potential function under NVT ensemble as described in section 3. The torsional elastic response of the $(10,10)$ SWCNT is depicted in Fig. 4. The torque (measured as the product of torsional force and the radius of the SWCNT) increases linearly with strain until about it reaches a specific torsional strain $\gamma=0.07$. After this point, we note that the nature of the curve deviates markedly from its linear behavior. This change in curve is caused due to the elastic to plastic transition of the SWCNT under torsion, which is in good agreement with results reported in earlier research [12]. It should further be noted from this figure that the observed maximum torque of 14.698 $\mathrm{nN} / \mathrm{nm}$ at torsional strain of $\gamma=0.078$ is in excellent agreement with the values reported in Ref. [15]. 


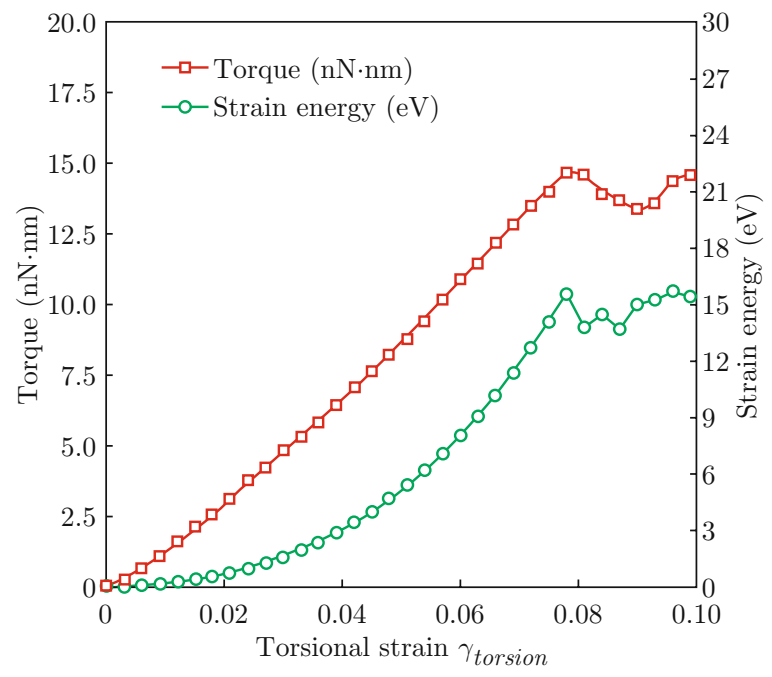

Fig. 4 Torque-strain and strain energy plot of the $(10,10)$ SWCNT of length, $L=100 \AA$ at $300 \mathrm{~K}$.

\section{Effect of surrounding water molecules}

This section focuses on the effect of surrounding water molecules on the torsional properties of SWCNT. This is accomplished by submerging a $(10,10)$ SWCNT in a simulation box filled with water molecules of density $0.9 \mathrm{~g} / \mathrm{cm}^{3}$. Three lengths of the SWCNT are considered here, viz. $L=50 \AA, 75 \AA$ and $100 \AA$. These lengths correspond to aspect ratios $(L / D)$ of $3.69,5.53$ and 7.37 respectively. It should be noted that we maintain the same aspect ratio for different SWCNTs considered in our study which will be described in the subsequent sections. Figure 5 shows the torsional characteristics of the free form and water submerged (10, 10) SWCNT. It can be noted from this figure that the maximum torque decreases while the torsional angle increases with the aspect ratio of the SWCNT, which is similar to results reported previously Ref. $[15,16]$. This is because SWCNTs with small aspect ratio are stocky
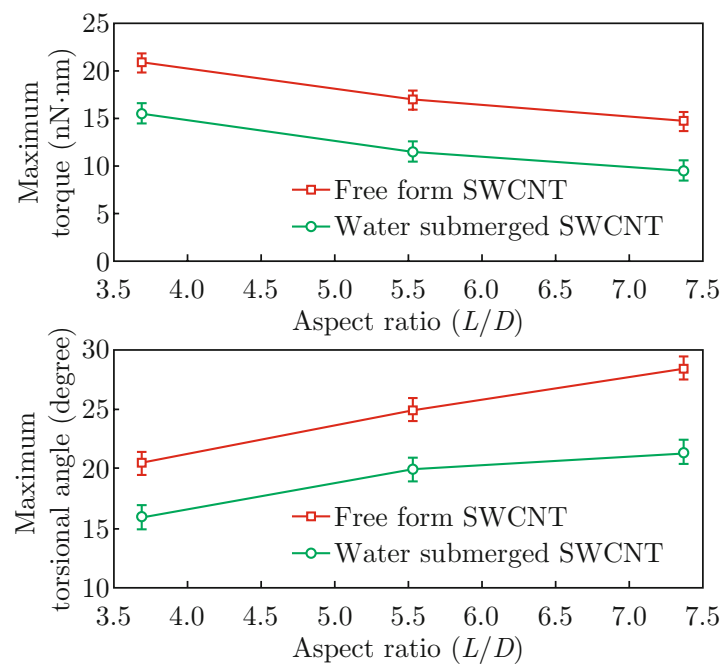

Fig. 5 Plot of the variation in the torsional response for free form and water submerged $(10,10)$ SWCNTs of different aspect ratios. and would require larger torque while SWCNTs with large aspect ratio are slender and can be twisted easily to large angles. Furthermore, this dependence is in agreement with the continuum elastic definition of torque $(T)$ given by Ref. [15],

$$
T=K \theta / L
$$

where $K$ is the torsional stiffness, $\theta$ is the torsional angle and $L$ is the length of the tube.

It can be noted from Fig. 5 that the effect of interacting water molecules decreases the torsional performance of the SWCNT. This can be explained as follows. The plot of the strain energy of the free form and water submerged SWCNT plotted against strain is illustrated in Fig. 6. It can be seen from this figure that the strain energy of water submerged SWCNT is higher than that of the free form SWCNT at smaller strain levels. The interaction of water molecules surrounding the SWCNT increases the strain on the SWCNT surface which reduces the torsional performance of the SWCNT. Additionally, we can also see from this figure that the presence of water molecules surrounding the SWCNT decreases the threshold strain energy required to induce elasticity to plastic transition in the SWCNT due to torsional twisting.

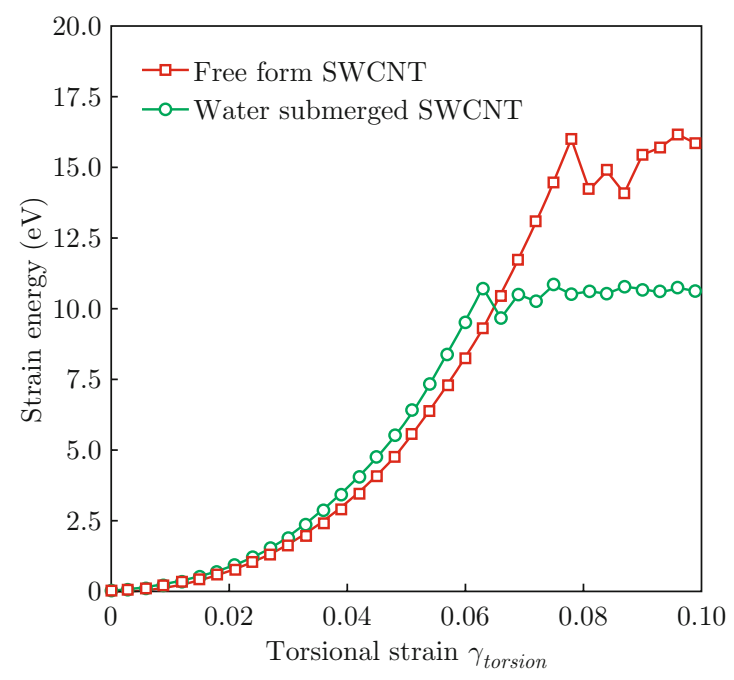

Fig. 6 Plot of the strain energy of the free form and water submerged $(10,10)$ SWCNT of aspect ratio $L / D=7.37$.

It is also worthwhile to note from Fig. 6 that at the onset of plastic deformation, there is a change in the quadratic behavior of the strain energy curve of free form and water submerged SWCNT with torsional strain. Thereafter, the strain energy of free form and water submerged SWCNT fluctuates slowly with increasing torsional angle in the plastic stage. This gradual change of the strain energy after plastic transition is different to the drastic drop in strain energy for the case of SWCNTs under tensile [30] or compressive loading $[20,36]$. This phenomenon as explained in Ref. [12] is due to the reason that the elastic to plastic transition 

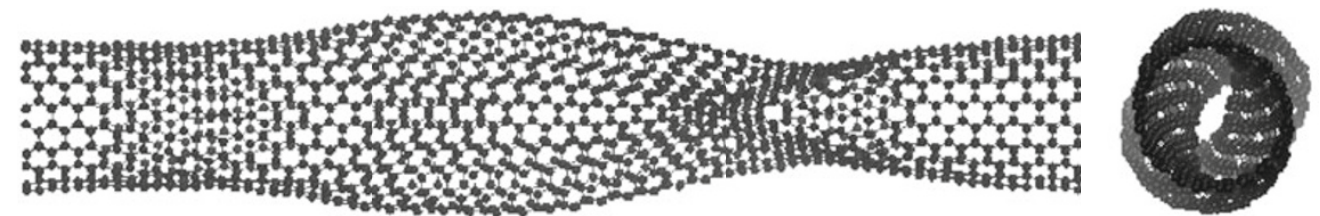

(a)
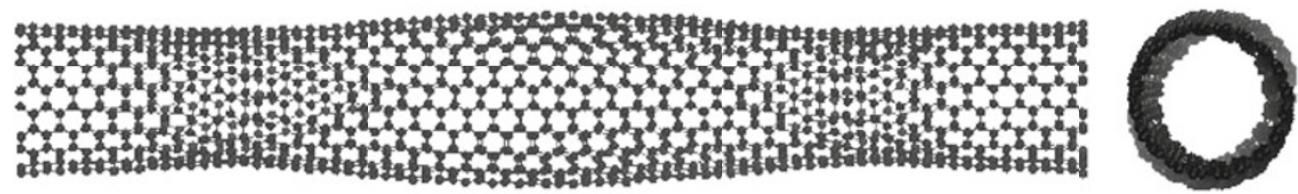

(b)
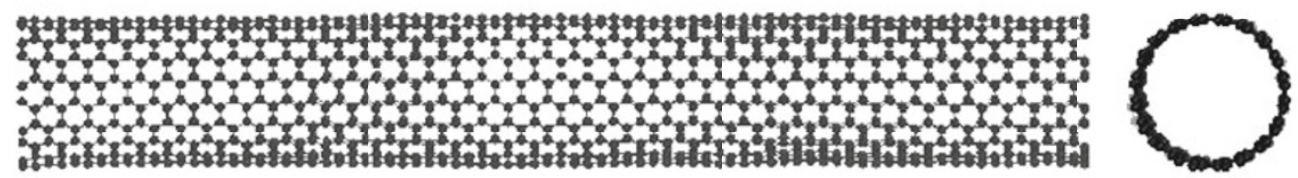

(c)
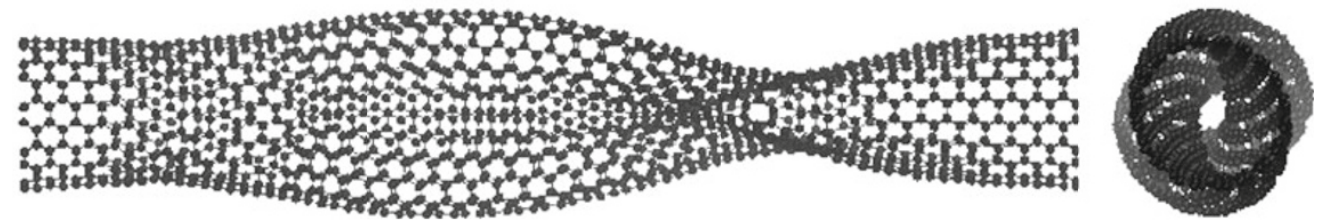

(d)

Fig. 7 Snapshots of torsional deformation of water submerged $(10,10)$ SWCNT of aspect ratio, $L / D=7.37$ when it is twisted by (a) $0^{\circ}$, (b) $30^{\circ}$, (c) $60^{\circ}$, and (d) $90^{\circ}$. The water molecules are not shown in this figure for clarity.

in an SWCNT under torsion results in a small structural change compared to that of the tensile or compressive loading. The variation in the strain energy fluctuations of free form and water submerged SWCNT in the plastic stage may be attributed to the difference in loading conditions on SWCNT caused due to absence and presence of water molecules respectively. The snap shots of the torsional deformation of water submerged SWCNTs are shown in Fig. 7. In this figure, only the carbon atoms comprising the SWCNT are shown and the water molecules surrounding the SWCNT have been made invisible for the ease of analysis. It can be seen from this figure that the SWCNT maintains its stable structure when no torsional twisting is applied at the end atoms of the SWCNT. When the end atoms of the SWCNT are subjected to the torsion of $30^{\circ}$, the resulting structure shows small changes compared to that of the initial SWCNT before torsion. Higher torsional angles results in screw twisted configurations of the SWCNT.

The radial distribution of water molecules surrounding a $(10,0)$ SWCNT submerged in the simulation box consisting of water molecules is shown in Fig. 8. It can be noted from this figure that the first peak occurs at small radial distances of SWCNT not exceeding $0.05 \mathrm{~nm}$. This means that the water molecules inside SWCNT are more concentrated near the center of the
SWCNT. We also see that the radial distribution of the first peak decreases while increasing the angle of twist. This is because twisting of SWCNT results in squeezing of water molecules from the interior of the SWCNT to outer space in simulation box. This could also be a possibility as to why we can see an increase in the second characteristic peak of water molecules outside

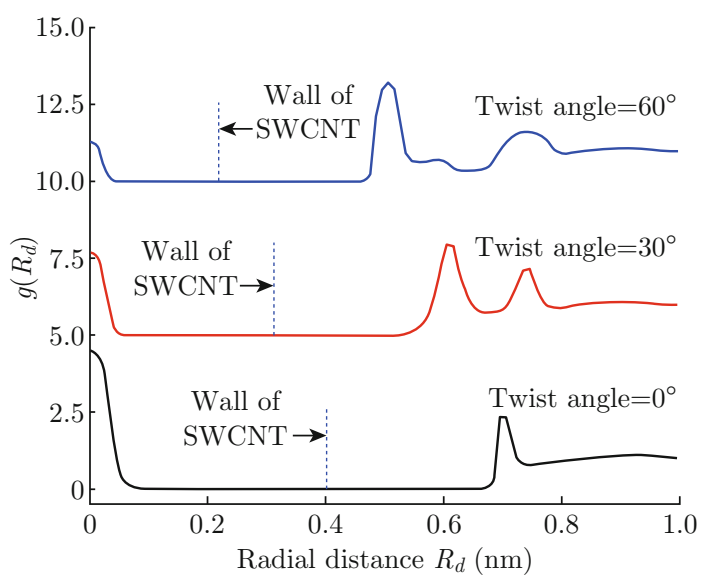

Fig. 8 RDF of water molecules during torsional loading of a $(10,0)$ SWCNT submerged in simulation box consisting of water molecules for different angles of twisting displaced vertically for clarity. The torsional loading of SWCNT results in wall contraction of the SWCNT. 

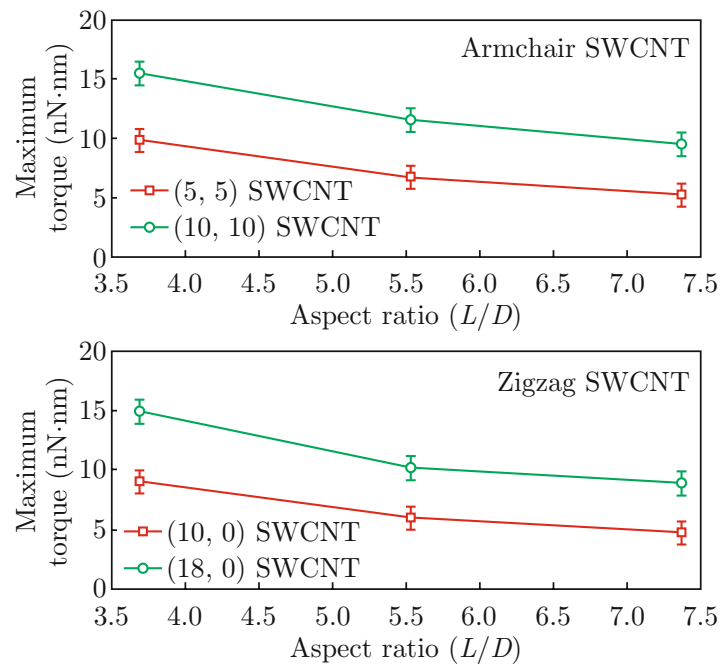

Fig. 9 Torsional characteristics of armchair and zigzag SWCNTs submerged in water at temperature $T=300 \mathrm{~K}$.

the wall of SWCNT. Similarly, as angle of twist increases, the occurrence of second characteristic peak also decreases. This is because the contraction of the SWCNT due to twisting increases van der Waal's distance between carbon and water molecules that will further push the water molecules towards the wall of the SWCNT.

\section{Effect of SWCNT geometry}

In this section we investigate the effect of SWCNT geometry on the torsional properties of SWCNT submerged in water. The effect of chirality is analyzed by investigating the torsional characteristics of armchair and zigzag SWCNT. The effect of size is analyzed by varying the aspect ratio of the considered SWCNTs. We employed $(10,10),(5,5),(18,0)$ and $(10,0)$ SWCNTs of three aspect ratios, viz. 3.69, 5.53 and 7.37. The SWCNTs are then submerged in a simulation box filled with water molecules of density $0.997 \mathrm{~g} / \mathrm{cm}^{3}$ (approx. $1 \mathrm{~g} / \mathrm{cm}^{3}$ ) and subjected to torsion. The variation in the torsional characteristics of the armchair and zigzag water submerged SWCNTs are shown in Fig. 9. We note from this figure that regardless of chirality, the torque decreases with reducing diameter of the SWCNT. This is due to the reason that reducing the diameter of SWCNT decreases its cross sectional area which ultimately lowers down the maximum torque of the SWCNT. Figure 9 also shows that the armchair water submerged SWCNTs can withstand slightly higher torque compared to that of zigzag SWCNTs. This observation is in quantitative agreement with the study on torsional properties of free form of SWCNTs reported by Zhang et al. [12]. It can be noted from Fig. 10 that the application of torsional loading in SWCNTs induces radial wall contraction of the SWCNT. The wall contraction of an armchair SWCNT is comparatively lower than that of zigzag SWCNT. The bonds in armchair
SWCNT are arranged parallel to the radial circumference of SWCNT which could result in better resistance to the torsional deformation.

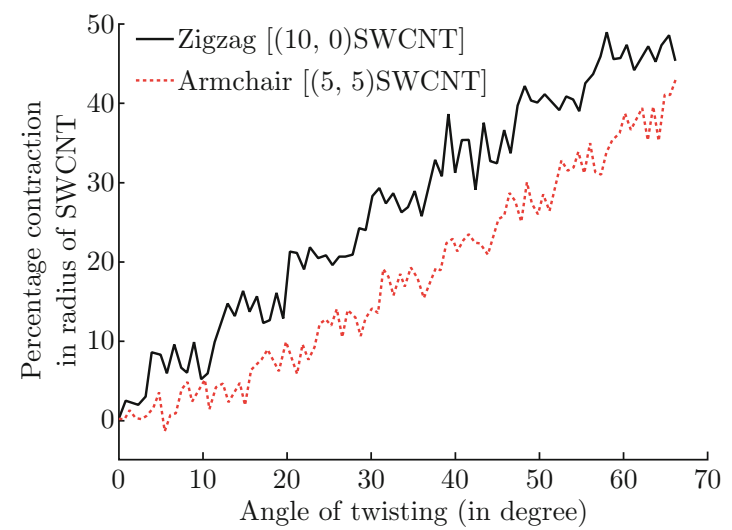

Fig. 10 Percentage contraction in radius of armchair and zigzag SWCNTs.

\section{Effect of location and number of defects on SWCNT}

The effect of location and number of defects on elastic properties of water submerged SWCNT is discussed in this section. For this purpose we considered $(5,5)$ and $(10,10)$ SWCNTs of aspect ratio 7.37 under torsional loading as shown in Fig. 1. The mechanical characteristics of the water submerged SWCNTs with different defect density are shown in Fig. 11. The presence of defects strongly impact the torsional properties of water submerged SWCNTs which is consistent with the observations on free form SWCNTs [12]. Additionally, it can be seen that the dependence of torque on the presence of vacancy defects is more sensitive to the diameter of SWCNT. For instance, the percentage drop in maximum torque for a $(5,5)$ SWCNT due to the presence of 3 vacancy defects in axial direction is about $41 \%$. This drop is significantly much higher than that of a $(10,10)$ SWCNT with similar vacancy configuration that experience a drop of about $16 \%$. This is because the $(5,5)$ SWCNT has a smaller diameter and the defect region occupies a much larger portion when compared to that of the $(10,10)$ SWCNT with a bigger diameter.

We also investigated the effect of defect location on the torsional response of water submerged SWCNTs. By varying the defects along the axial or radial direction of SWCNT (Fig. 2), the dependence of defect distribution on the torsional response can be studied. We note from Fig. 11 that the defects placed along the radial direction of SWCNT has a significant impact on the torsional response of SWCNT compared to the axial defects. Defects along the radial direction are arranged parallel to the loading direction, which will aid in breaking and shear of SWCNTs more easily in contrast to that of the axial defects that are perpendicular 
to the torsional loading direction. This is an important factor that needs to be considered while designing SWCNT based fluid delivery systems. Adequate care must be taken to ensure that the radial defects are kept to the minimum to ensure greater stability of SWCNT based nano-fluidic devices.
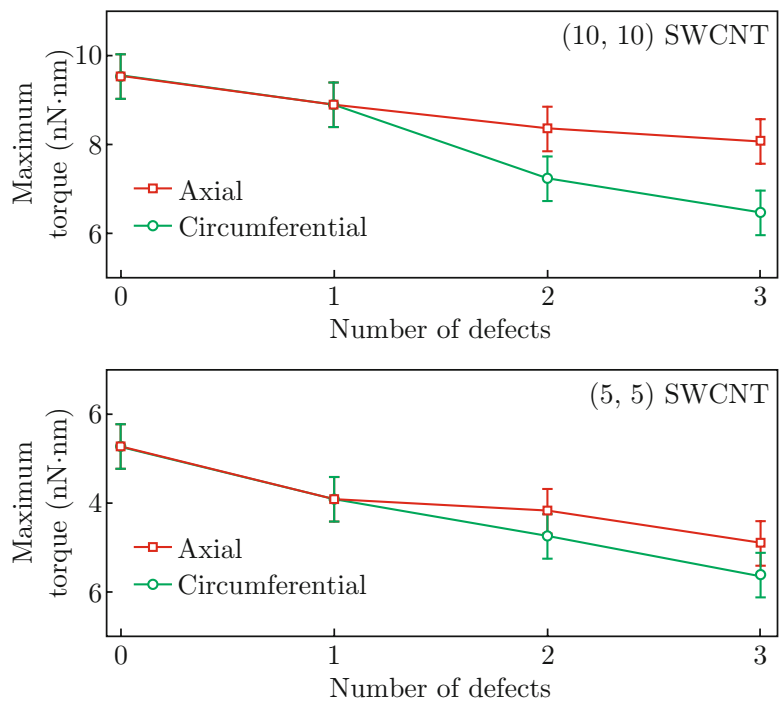

Fig. 11 Variation of mechanical properties of $(10,10)$ and $(5,5)$ SWCNTs of aspect ratio $L / D=7.37$ with increasing the vacancy defect concentration along the axial and circumferential direction.

\section{Analysis of SWCNT torsion with continuum me- chanics theory}

The torsional stiffness $K$ of the SWCNTs in our study is computed in MD simulation method by obtaining the variation of strain energy $E$ with the torsional angle $\theta$. The torsional stiffness is then defined by the following equation $[11,15]$

$$
K=L \frac{d^{2} E}{d \theta^{2}}
$$

where $L$ is the length of the SWCNT.

The torsional stiffness of the free form and water submerged $(10,10)$ and $(5,5)$ SWCNTs of aspect ratio 7.37 is computed using Eq. (7). The results are then plotted against the respective radius of the $(10,10)$ and $(5,5)$ SWCNTs and is presented in Fig. 12. It can be noted from this figure that the torsional stiffness varies marginally due to the presence of surrounding water molecules. This observation is consistent with the results reported in earlier studies of Ref. $[16,17]$ for the case of SWCNTs interacting with various gases and metals. For the case of freeform SWCNTs, the torsional stiffness of $(10,10)$ SWCNT has increased by $670 \%$ compared to that of $(5,5)$ SWCNT. Similar percentage variations can also be observed for the case of $(10,10)$ and $(5,5)$ SWCNTs that are submerged in water.
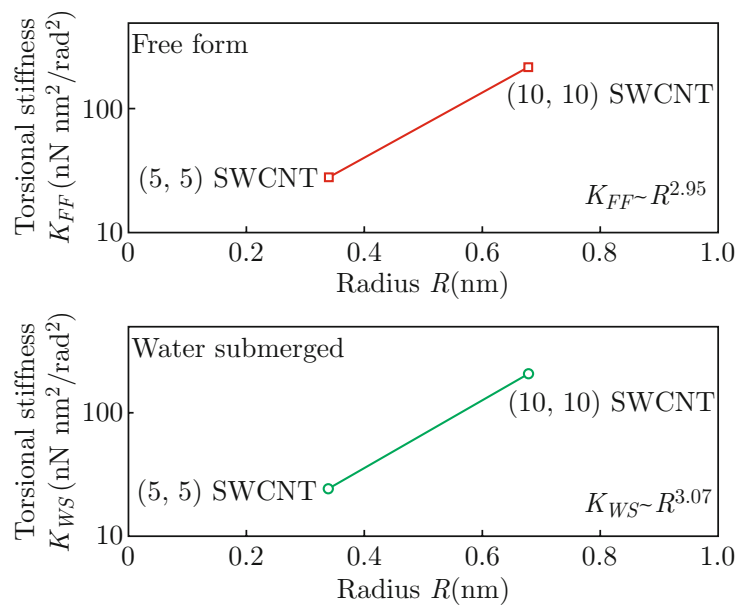

Fig. 12 Torsional stiffness of free form $\left(K_{F F}\right)$ and water submerged $\left(K_{W S}\right)$ SWCNTs depicted for various radius of SWCNTs considered in our study.

The torsional stiffness $K$ is heavily dependent on the radius, $R$ of the SWCNT. It shows a relation of $K_{F F} \sim R^{2.95}$ and $K_{W S} \sim R^{3.07}$ where the subscripts 'FF' and 'WS' refers to 'free form' and 'water submerged' states of SWCNTs respectively (Fig. 12). It is worthy to note that the variation is consistent with the prediction of linear elasticity in continuum mechanics theory. In continuum mechanics theory, the strain energy of a thin walled cylinder is defined as $[11,15]$

$$
E=\frac{1}{2} G_{\text {torsion }} \int_{V} \gamma^{2} d V=G_{\text {torsion }} \pi h R_{c y l}^{3} \frac{\theta^{2}}{L}
$$

where $G_{\text {torsion }}$ is the torsional modulus, $\gamma$ is the torsional strain, $V$ is the volume of the cylinder, $\theta$ is the critical torsional angle, $h$ and $R_{c y l}$ describes the thickness and radius of the cylinder respectively.

The torsional stiffness of the thin walled cylinder is computed from Eq. (7) and Eq. (8) and is expressed as

$$
K=L \frac{d^{2} E}{d \theta^{2}}=2 G_{\text {torsion }} \pi h R_{c y l}^{3}
$$

From Eq. (9) we can see that the torsional stiffness of the thin walled cylinder varies as $K \sim R_{c y l}^{3}$, which is in good agreement with the relation $\left(K_{F F} \sim R^{2.95}\right.$ and $K_{W S} \sim R^{3.07}$ ) obtained using the MD simulation approach.

The torsional modulus $G_{\text {torsion }}$ of SWCNT can be obtained from the quadratic plot of variation of strain energy $E$ with torsional strain $\gamma$ using polynomial curve fitting, which is defined as $[11,37]$,

$$
G=\frac{1}{2 \pi R L h} \frac{\partial^{2} E}{\partial \gamma^{2}}
$$

where $R$ is the radius of the SWCNT, $h$ is the thickness of SWCNT and is assumed to be $3.4 \AA$ [38].

The computed torsional modulus of various free form and water submerged SWCNTs of aspect ratio 7.37 under torsion is depicted in Table 2. It can be observed 
that the interaction due to surrounding water molecules decreases the torsional modulus of SWCNTs. It is important to note that the trend of torsional modulus variation for armchair and zigzag SWCNTs is agree well with the earlier results obtained by MD simulation studies $[10,12,15]$. However, the computed torsional modulus is lesser than the values predicted using continuum theory [39] and experiment [9]. The corresponding shear modulus values obtained in these literature studies is given in Table 3.

Table 2 Torsional modulus of freeform and water submerged SWCNTs

\begin{tabular}{cccc}
\hline Geometry & $\begin{array}{c}\text { Free form } \\
\text { SWCNT } \\
(\mathrm{TPa})\end{array}$ & $\begin{array}{c}\text { Water submerged } \\
\text { SWCNT (TPa) }\end{array}$ & $\begin{array}{c}\text { Percentage } \\
\text { variation } \\
(\%)\end{array}$ \\
\hline$(10,10)$ & 0.378 & 0.351 & 7.1 \\
$(5,5)$ & 0.335 & 0.304 & 9.2 \\
$(18,0)$ & 0.367 & 0.338 & 7.9 \\
$(10,0)$ & 0.329 & 0.302 & 8.2 \\
\hline
\end{tabular}

Table 3 A summary of torsional modulus of SWCNTs reported in literature

\begin{tabular}{lll}
\hline Authors & $\begin{array}{l}\text { Employed } \\
\text { technique }\end{array}$ & $\begin{array}{l}\text { Torsional } \\
\text { modulus } \\
(\mathrm{TPa})\end{array}$ \\
\hline Zhang et al. [12] & MD simulation & 0.381 \\
Jeong et al. [15] & MD simulation & 0.338 \\
Wang et al. [10] & MD simulation & $0.36 \sim 0.48$ \\
Lu and Zhang [39] & Nanoscale continuum & $0.41 \sim 0.54$ \\
& $\quad$ theory & \\
Hall et al. [9] & Experimental method & $0.41 \pm 0.36$ \\
Present authors & MD simulation & $0.329 \sim 0.378$ \\
\hline
\end{tabular}

\section{Elastic properties of capped SWCNT}

The torsional properties of capped SWCNTs encapsulated with water molecules is discussed in this section. For this purpose, we chose a $(10,10)$ capped SWCNT with aspect ratio of 7.37 encapsulated with water molecules of three different densities $(\rho)$ viz. empty $\left(\rho=0 \mathrm{~g} / \mathrm{cm}^{3}\right)$, half-filled $\left(\rho=0.49 \mathrm{~g} / \mathrm{cm}^{3}\right)$ and fully-filled $\left(\rho=0.98 \mathrm{~g} / \mathrm{cm}^{3}\right)$ SWCNT. The torsional properties of such water encapsulated SWCNTs were then investigated in free form and water submerged states. The torsional response of the free form capped SWCNTs with water molecules of different encapsulation densities is shown in Fig. 13. We note from this figure that the torsional characteristics of half-filled SWCNTs are almost similar to that of an empty SWCNT. The interaction force exerted by water molecules on the half-filled capped SWCNT is depicted in Fig. 14. It can be seen from this plot that the water molecules exert an average of $1.18 \mathrm{nN} / \mathrm{nm}$, which is insignificant to

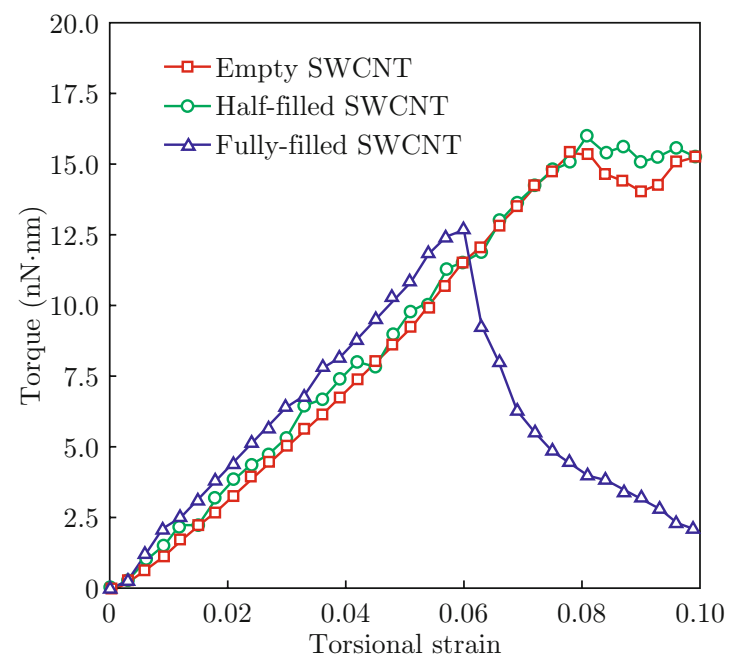

Fig. 13 Torsional response of a capped $(10,10)$ SWCNT with aspect ratio $(L / D)$ of 7.37 with varying water concentrations encapsulated inside the SWCNT.

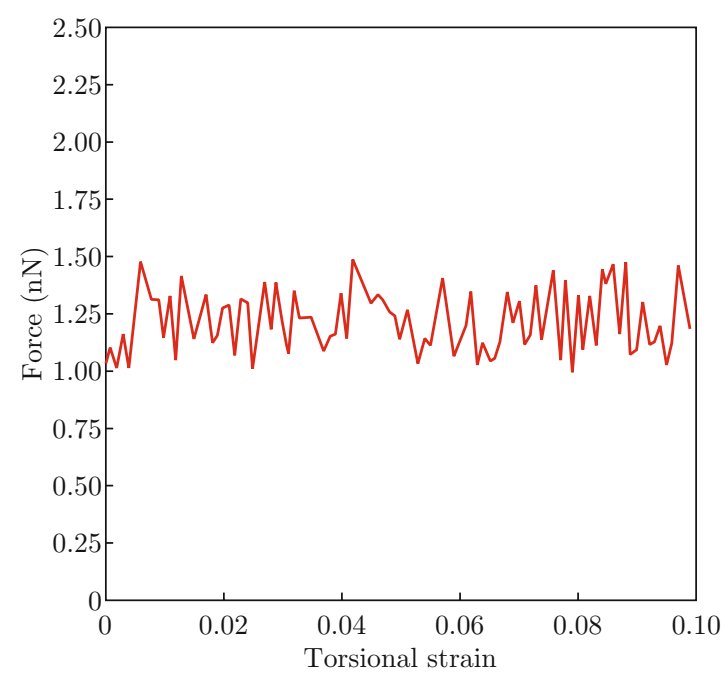

Fig. 14 Interaction force exerted by water molecules on an $(10,10)$ SWCNT encapsulated with water molecules of density, $\rho=0.45 \mathrm{~g} / \mathrm{cm}^{3}$.

cause any variation in the torsional response of halffilled SWCNT. Additionally, for the case of a fully-filled SWCNT, the torsional response shows a marked difference compared to that of the empty and half-filled SWCNTs. It can be noted from Fig. 13 that prior to torsion, the fully-filled SWCNT can withstand more torque compared to that of an empty and half-filled SWCNT. This observation is consistent with the earlier reports of the torsional response of SWCNTs filled with SWCNTs [15], gases [17] and metals [16]. It is worth noting that when torsional strain is about 0.06 , there is a sudden drop in torque of a fully-filled SWCNT in contrast to that of the empty and half-filled SWCNTs. This can be explained by analyzing the snap shot of the torsional deformation of fully-filled SWCNTs as depicted in Fig. 15. The torsional twisting of SWCNT 

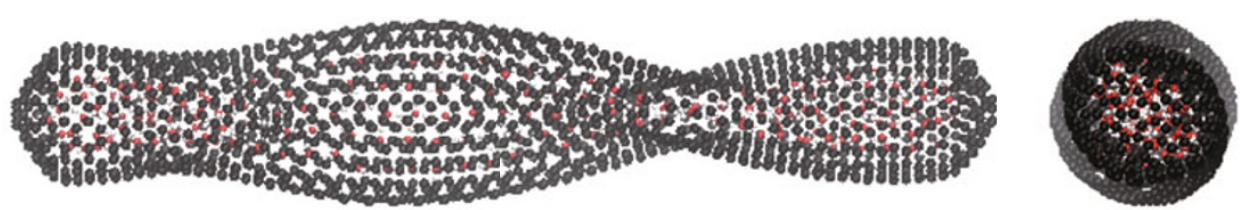

(a)
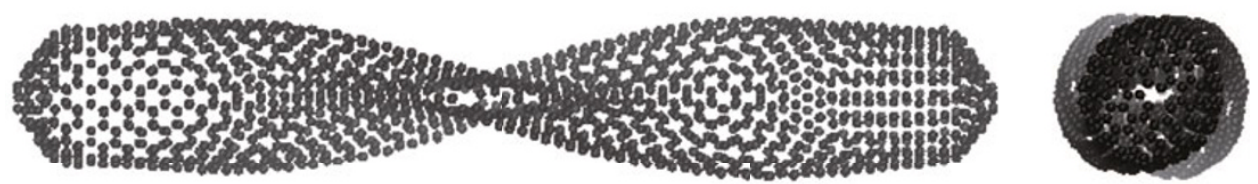

(b)
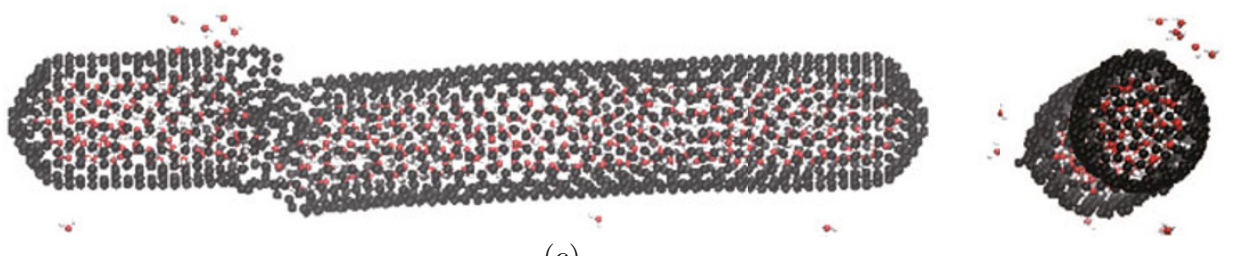

(c)

Fig. 15 Snap shot of the post twisted shapes exhibited by capped SWCNTs encapsulated with water molecules of varying densities. It can be observed that an (a) empty SWCNT and (b) half-filled SWCNT do not fracture when it is subjected to torsion. However a (c) fully-filled SWCNT breaks in response to torsion.
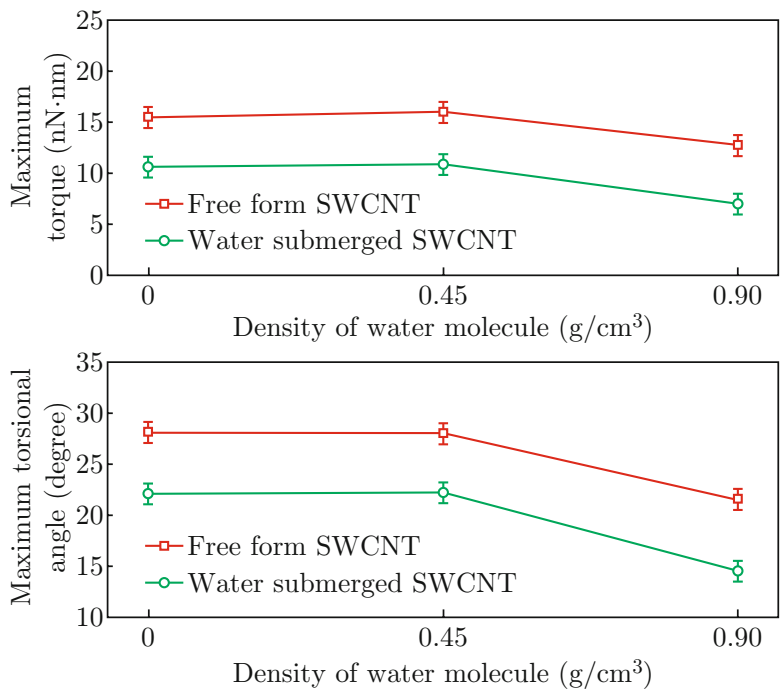

Fig. 16 Torsional properties of the capped $(10,10)$ SWCNTs filled with varying water concentrations submerged in water at temperature $T=300 \mathrm{~K}$.

results in contraction of tube walls in the radial direction of the SWCNT (as shown in Fig. 10). This contraction result is in compression of water molecules. Because we cannot compress the water molecules further, therefore the water molecules have nowhere to escape, but to break open the walls of SWCNT which results in sudden drop in the torsional response of the fully-filled SWCNT. Additionally, the release of encapsulated water molecules from the fully-filled SWCNT due to torsional loading can prove to be a useful mechanism for nano-level fluidic devices aimed at targeted drug-delivery.
It is also useful to investigate the torsional response of water-encapsulated capped SWCNTs submerged in water. The variation in the torsional response of the water submerged capped SWCNTs are compared with that of the free form SWCNTs as shown in Fig. 16. Figure 16 indicates that the effect of surrounding water molecules reduces the torsional properties of water encapsulated SWCNTs. Based on our foregoing discussion, presence of surrounding water molecules increases the strain of the capped SWCNT which affects the torsional resistance of capped SWCNT. Hence, it can be concluded from this study that dense packing of water molecules can result in breaking and fracture of capped SWCNTs. Furthermore, the interaction of water molecules outside the capped SWCNT can affect the overall torsional response of capped SWCNTs irrespective of the filling concentrations.

\section{Conclusions}

In this paper, the torsional characteristics of water submerged SWCNT has been studied using MD simulation method. The understanding of torsional properties of SWCNT in hydrodynamics environment is crucial for optimizing the performance of nano-fluidic based NEMS devices. The studies show that the torsional characteristics of SWCNTs reduce due to the interaction of surrounding water molecules. The results also indicate that the torsional properties of SWCNTs with defects are more sensitive to the defect distribution and diameter of the SWCNT. Furthermore, for the case of capped SWCNTs, dense filling of water molecules in an 
SWCNT will result in breaking and fracture of SWCNT under torsion resulting in release of encapsulated water molecules. This mechanism could be useful in the design of SWCNT based nano-drug delivery devices. The results obtained from our studies will provide valuable insights for the application of CNTs for nano-level processes involving water molecules such as nano-fluidic devices, NEMS, and drug delivery devices.

\section{References}

[1] M. Liu, Z. Wu, W. M. Lau and J. Yang, "Recent advances in directed assembly of nanowires or nanotubes", Nano-Micro Lett. 4(3), 142-153 (2012). http://dx.doi.org/10.3786/nml.v4i3.p142-153

[2] V. Vijayaraghavan and C. H. Wong, "Shear deformation characteristics of single walled carbon nanotube with water interactions by using molecular dynamics simulation", Phys. E: LowDimensional Systems and Nanostructures 54(0), 206213 (2013). http://dx.doi.org/http://dx.doi.org/ 10.1016/j.physe.2013.06.025

[3] P. A. Williams, S. J. Papadakis, A. M. Patel, M. R. Falvo, S. Washburn and R. Superfine, "Fabrication of nanometer-scale mechanical devices incorporating individual multiwalled carbon nanotubes as torsional springs", Appl. Phys. Lett. 82(5), 805-807 (2003). http://dx.doi.org/10.1063/1.1538346

[4] Y. Zhang, L. F. Duan, J. Wang, H. Geng and Q. Zhang, "Advances in conceptual electronic nanodevices based on $0 \mathrm{D}$ and $1 \mathrm{D}$ nanomaterials", Nano-Micro Lett. 6(1), 1-19 (2014). http://dx.doi.org/10.5101/ nml.v6i1.p1-19

[5] Y. Cheng, Q. X. Pei and H. J. Gao, "Moleculardynamics studies of competitive replacement in peptide-nanotube assembly for control of drug release", Nanotechnology 20(14), 145101 (2009). http://dx.doi.org/10.1088/0957-4484/20/14/ 145101

[6] D. Xu, Z. Hu, J. Su, F. Wu and W. Yuan, "Micro and nanotechnology for intracellular delivery therapy protein", Nano-Micro Lett. 4(2), 118-123 (2012). http:// dx.doi.org/10.3786/nml.v4i2.p118-123

[7] X. Sun, L. Qiao and X. Wang, "A novel immunosensor based on Au nanoparticles and polyaniline/multiwall carbon nanotubes/ chitosan nanocomposite film functionalized interface", Nano-Micro Lett. 5(3), 191201 (2013). http://dx.doi.org/10.5101/nml.v5i3. p191-201

[8] D. R. Shobha Jeykumari, R. A. Kalaivani and S. Sriman Narayanan, "Nanobiocomposite electrochemical biosensor utilizing synergic action of neutral red functionalized carbon nanotubes", Nano-Micro Lett. 4(4), 220-227 (2012). http://dx.doi.org/10.3786/ nml.v4i4.p220-227

[9] A. R. Hall, L. An, J. Liu, L. Vicci, M. R. Falvo, R. Superfine and S. Washburn, "Experimental measurement of single-wall carbon nanotube torsional proper- ties", Phys. Rev. Lett. 96(25), 256102 (2006). http:// $\mathrm{dx}$.doi.org/10.1103/PhysRevLett.96.256102

[10] Y. Wang, X. X. Wang and X. G. Ni, "Atomistic simulation of the torsion deformation of carbon nanotubes", Modelling Simul. Mater. Sci. Eng. 12(6), 1099-1107 (2004). http://dx.doi.org/10.1088/0965-0393/12/ $6 / 004$

[11] Y. Y. Zhang, C. M. Wang and V. B. C. Tan, "Mechanical Properties and Buckling Behaviors of Condensed Double-Walled Carbon Nanotubes", J. Nanosci. Nanotechnol. 9(8), 4870-4879 (2009). http://dx.doi.org/ 10.1166/jnn.2009.1092

[12] Y. Y. Zhang, C. M. Wang and Y. Xiang, "A molecular dynamics investigation of the torsional responses of defective single-walled carbon nanotubes", Carbon. 48(14), 4100-4108 (2010). http://dx.doi.org/ $10.1016 / j$.carbon. 2010.07.018

[13] X. X. Lu and Z. Hu, "Mechanical property evaluation of single-walled carbon nanotubes by finite element modeling", Composites Part B-Engineering. 43(4), 1902-1913 (2012). http://dx.doi.org/10. 1016/j. compositesb. 2012.02.002

[14] B. Faria, N. Silvestre and J. N. C. Lopes, "Induced anisotropy of chiral carbon nanotubes under combined tension-twisting", Mech. Mater. 58, 97109 (2013). http://dx.doi.org/10.1016/j.mechmat. 2012.11.004

[15] B. W. Jeong, J. K. Lim and S. B. Sinnott, "Elastic torsional responses of carbon nanotube systems", J. Appl. Phys. 101(8), 084309 (2007). http://dx.doi.org/10. 1063/1. 2717138

[16] L. Wang, Z. Q. Zhang, Y. G. Zheng, J. B. Wang and H. F. Ye, "Torsional stability of carbon nanotubes filled with copper atoms", Phys. Scripta. 85(4), 045602 (2012). http://dx.doi.org/10.1088/0031-8949/85/ $04 / 045602$

[17] Q. Wang, K. M. Liew and V. K. Varadan, "Molecular dynamics simulations of the torsional instability of carbon nanotubes filled with hydrogen or silicon atoms", Appl. Phys. Lett. 92(4), 043120 (2008). http://dx. doi.org/10.1063/1.2840165

[18] S. Plimpton, "Fast Parallel Algorithms for ShortRange Molecular Dynamics", J. Comput. Phys. 117(1), 1-19 (1995). http://dx.doi.org/10.1006/ jcph. 1995. 1039

[19] S. J. Stuart, A. B. Tutein and J. A. Harrison, "A reactive potential for hydrocarbons with intermolecular interactions", J. Chem. Phys. 112(14), 6472-6486 (2000). http://dx.doi.org/10.1063/1.481208

[20] Y. Y. Zhang, C. M. Wang and V. B. C. Tan, "Buckling of carbon nanotubes at high temperatures", Nanotechnology. 20(21), 215702 (2009). http://dx.doi. org/10.1088/0957-4484/20/21/215702

[21] C. H. Wong and V. Vijayaraghavan, "Nanomechanics of free form and water submerged single layer graphene sheet under axial tension by using molecular dynamics simulation", Mater. Sci. Eng. A 556, 420-428 (2012). http://dx.doi.org/10.1016/j.msea.2012.07.008 
[22] C. H. Wong and V. Vijayaraghavan, "Nanomechanics of Nonideal Single- and Double-Walled Carbon Nanotubes", J. Nanomater. 2012, 17 (2012). http://dx . doi.org/10.1155/2012/490872

[23] D. W. Brenner, O. A. Shenderova, J. A. Harrison, S. J. Stuart, B. Ni and S. B. Sinnott, "A second-generation reactive empirical bond order (REBO) potential energy expression for hydrocarbons", J. Phys.-Conden. Matt. 14(4), 783-802 (2002). http://dx.doi.org/10. 1088/0953-8984/14/4/312

[24] Y. Nakamura and T. Ohno, "Structure of water confined inside carbon nanotubes and water models", Mater. Chem. Phys. 132(2-3), 682-687 (2012). http:// dx. doi .org/10.1016/j . matchemphys. 2011.11.086

[25] C. H. Wong and V. Vijayaraghavan, "Compressive characteristics of single walled carbon nanotube with water interactions investigated by using molecular dynamics simulation", Phys. Lett., Sect. A 378(5), 570-576 (2014). http://dx.doi.org/10.1016/ j.physleta. 2013.12.026

[26] T. Werder, J. H. Walther, R. L. Jaffe, T. Halicioglu and P. Koumoutsakos, "On the water-carbon interaction for use in molecular dynamics simulations of graphite and carbon nanotubes", J. Phys. Chem. B 107(6), 1345-1352 (2003). http://dx.doi.org/10. 1021/jp0268112

[27] N. Marković P. U. Andersson, M. B. Någård and J. B. C. Pettersson, "Scattering of water from graphite: simulations and experiments", Chem. Phys. 247(3), 413-430 (1999). http:/dx.doi.org/10.1016/ S0301-0104 (99) 00233-5

[28] X. B. Zhang, Q. L. Liu and A. M. Zhu, "An improved fully flexible fixed-point charges model for water from ambient to supercritical condition", Fluid Phase Equilibria. 262(1-2), 210-216 (2007). http://dx.doi.org/ 10.1016/j.fluid.2007.09.005

[29] C. H. Wong, "Elastic properties of imperfect singlewalled carbon nanotubes under axial tension", Comput. Mater. Sci. 49(1), 143-147 (2010). http://dx. doi.org/10.1016/j.commatsci. 2010.04.037

[30] V. Vijayaraghavan and C. H. Wong, "Nanomechanics of single walled carbon nanotube with water interactions under axial tension by using molecular dynamics simulation", Comput. Mater. Sci. 79, 519-526 (2013). http://dx.doi.org/10.1155/2012/490872

[31] W. G. Hoover, "Canonical dynamics - equilibrium phase-space distributions", Phys. Rev. A 31(3), 16951697 (1985). http://dx.doi.org/10.1103/PhysRevA. 31.1695

[32] S. Nose, "A unified formulation of the constant temperature molecular-dynamics methods", J. Chem. Phys 81(1), 511-519 (1984). http://dx.doi.org/ http://dx.doi.org/10.1063/1.447334

[33] L. Verlet, "Computer 'Experiments' on Classical Fluids. I. Thermodynamical Properties of Lennard-Jones Molecules", Phys. Rev 159(1), 98-103 (1967). http:// dx.doi.org/10.1103/PhysRev. 159.98

[34] M. E. Suk and N. R. Aluru, "Water Transport through Ultrathin Graphene", J. Phys. Chem. Lett. 1(10), 1590-1594 (2010). http://dx.doi.org/ 10.1021/jz100240r

[35] Y. Homma, S. Chiashi, T. Yamamoto, K. Kono, D. Matsumoto, J. Shitaba and S. Sato, "Photoluminescence Measurements and Molecular Dynamics Simulations of Water Adsorption on the Hydrophobic Surface of a Carbon Nanotube in Water Vapor", Phys. Rev. Lett. 110(15), 157402 (2013). http://dx.doi.org/10. 1103/PhysRevLett. 110.157402

[36] V. Vijayaraghavan and C. H. Wong, "Temperature, defect and size effect on the elastic properties of imperfectly straight carbon nanotubes by using molecular dynamics simulation", Comput. Mater. Sci. 71, 184-191 (2013).

[37] K. Min and N. R. Aluru, "Mechanical properties of graphene under shear deformation", Appl. Phys. Lett. 98 (1), 013113 (2011). http://dx.doi.org/10.1063/ 1.3534787

[38] K. M. Liew, X. Q. He and C. H. Wong, "On the study of elastic and plastic properties of multiwalled carbon nanotubes under axial tension using molecular dynamics simulation", Acta Mater. 52(9), 2521-2527 (2004). http://dx.doi.org/10. $1016 / \mathrm{j}$. actamat . 2004.01 .043

[39] H. Lu and L. Zhang, "Analysis of localized failure of single-wall carbon nanotubes", Comput. Mater. Sci. 35(4), 432-441 (2006). http://dx.doi.org/10.1016/ j.commatsci.2005.02.011 